\title{
Governança Corporativa e Governança de Tecnologia da Informação como vantagem competitiva nas organizações
}

\author{
Corporate Governance and Information Technology Governance as a competitive advantage in
} organizations

El Gobierno Corporativo y el Gobierno de las Tecnologías de la Información como ventaja competitiva en las organizaciones

\author{
Adriana Maria Miguel Peixe \\ ORCID: https://orcid.org/0000-0003-0704-4076 \\ Universidade Federal do Paraná, Brasil \\ E-mail: ammp5196@gmail.com \\ Jorge Balsan \\ ORCID: https://orcid.org/0000-0002-9280-7797 \\ Universidade Federal do Paraná, Brasil \\ E-mail: balsan@hotmail.com \\ José Simão de Paula Pinto \\ ORCID: https://orcid.org/0000-0002-5023-437X \\ Universidade Federal do Paraná, Brasil \\ E-mail: simao@ufpr.br
}

\begin{abstract}
Resumo
A Tecnologia da Informação fundamentada em seus resultados e necessidades de utilização, possui no contexto atual, um cenário oportuno e relevante. Ela torna-se fundamental nas estratégias das empresas, explorando oportunidades e gerando vantagens competitivas em todos os níveis de gestão. Seus impactos são notórios nos níveis internos e externos das organizações: na estrutura orgânica e no papel de enquadramento/coordenação na organização; a nível psico-sociológico e das relações pessoais; no subsistema de objetivos e valores das pessoas que trabalham nas organizações; bem como no subsistema tecnológico. Neste contexto, o estudo teve como objetivo enfatizar o framework (estrutura) praticado e sua importância no contexto das vantagens competitivas nas organizações. Para atender à crescente demanda por inovação, criatividade, qualidade, melhoria de processos, competitividade dentre tantas outras necessidades neste mundo globalizado. Como delineamento metodológico, o artigo caracteriza-se como uma pesquisa bibliográfica, exploratória, documental, descritiva, explicativa e qualitativa. Infere-se que a janela de vantagem (período de tempo que leva para os concorrentes alcançar e igualar a vantagem estraté gica) tornou-se questão crítica em termos de gerenciamento. O desafio é encontrar as conexões gerenciais entre a TI, as soluções propostas e necessárias de tecnologia de informação, que atendam às necessidades de negócios, e os objetivos do negócio.
\end{abstract}

Palavras-chave: Governança corporativa; Governança de tecnologia da informação; Vantagem competitiva; Organizações.

\begin{abstract}
Information Technology, based on its results and usage needs, has a timely and relevant scenario in the current context. It becomes fundamental in companies' strategies, exploring opportunities and generating competitive advantages at all levels of management. Its impacts are notorious at the internal and external levels of organizations: on the organic structure and the role of framing / coordination in the organization; the psycho-sociological level and personal relationships; in the subsystem of objectives and values of the people who work in the organizations; as well as in the technological subsystem. In this context, the study aimed to emphasize the framework (structure) practiced and its importance in the context of competitive advantages in organizations. To meet the growing demand for innovation, creativity, quality, process improvement, competitiveness among many other needs in this globalized world. As a methodological design, the article is characterized as a bibliographic, exploratory, documentary, descriptive, explanatory and qualitative research. It is inferred that the window of advantage (the period of time it takes competitors to reach and match the strategic advantage) has become a critical issue in terms of management. The challenge is to find the managerial connections between IT, the proposed and necessary information technology solutions, which meet the business needs, and the business objectives.
\end{abstract}

Keywords: Corporate governance; Information technology governance; Competitive advantage; Organizations. 


\begin{abstract}
Resumen
La Tecnología de la Información con base en sus resultados y necesidades de uso, tiene un escenario oportuno y relevante en el contexto actual. Se vuelve fundamental en las estrategias de las empresas, explorando oportunidades y generando ventajas competitivas en todos los niveles de gestión. Sus impactos son notorios a nivel interno y externo de las organizaciones: en la estructura orgánica y en el rol de encuadre / coordinación en la organización; el nivel psicosociológico y las relaciones personales; en el subsistema de objetivos y valores de las personas que laboran en las organizaciones; así como en el subsistema tecnológico. En este contexto, el estudio tuvo como objetivo enfatizar el marco (estructura) practicado y su importancia en el contexto de las ventajas competitivas en las organizaciones. Satisfacer la creciente demanda de innovación, creatividad, calidad, mejora de procesos, competitividad entre muchas otras necesidades en este mundo globalizado. Como diseño metodológico, el artículo se caracteriza por ser una investigación bibliográfica, exploratoria, documental, descriptiva, explicativa y cualitativa. Se infiere que la ventana de ventaja (el período de tiempo que le toma a los competidores alcanzar e igualar la ventaja estratégica) se ha convertido en un tema crítico en términos de gestión. El desafío es encontrar las conexiones gerenciales entre TI, las soluciones de tecnología de la información propuestas y necesarias, que satisfagan las necesidades comerciales y los
\end{abstract} objetivos comerciales.

Palabras clave: Gobierno Corporativo; Gobernanza de la tecnología de la información; Ventaja competitiva; Organizaciones.

\title{
1. Introdução
}

A revolução da Informação exige, mudanças no modo como percebe-se a sociedade na organização e sua estrutura, o que se traduz num grande desafio: aproveitar as oportunidades, dominando os riscos inerentes ou submeter-se aos riscos com todas as incertezas que acarretam.

Para Nishioka; Przeybilovicz e Cunha (2014), o crescimento do segmento de TI tem revolucionado as organizações numa velocidade impressionante pelos espaços que são preenchidos, com o poder da inovação e da criação refletindo na aplicação de novas tecnologias em todo o mundo. Estar-se na atualidade conforme os autores (Silva; Araújo; Brito, 2018; Ferreira; Brito, 2020; Nascimento; Brito, 2020) inseridos em um ambiente mercadológico, competitivo e mutável. Adotar práticas, padrões e relacionamentos estruturados (executivos, gestores, técnicos e usuários de TI), torna-se fundamental. Para Ogécime; Silva, (2019), por trás desses operadores de transformação, as forças coexistem, repelem e se fundem umas com as outras.

A governança corporativa e governança de tecnologia da informação como vantagem competitiva nas organizações garantem, otimizar, ampliar e suportar as necessidades impostas pelo mercado competitivo, (Nishioka; Przeybilovicz \& Cunha 2014). Qualquer estratégia tem a tecnologia como forma de sustentabilidade.

A Tecnologia da Informação (TI) tem sido tratada por alguns grupos de discussão como estratégica para a competitividade das empresas, ou seja, a tecnologia da informação é um recurso estratégico para muitas empresas. Não há gestão (operacional, gerencial e estratégica) sem informações em tempo real e estas, fundamentais para a tomada de decisão. A informação é um elemento essencial para a sobrevivência humana seja no passado, presente e futuro.

O conceito de informação deriva do latim e significa um processo de comunicação ou algo relacionado com comunicação conforme Zhang, (1988), mas na realidade existem muitas e variadas definições de informação, cada uma mais complexa que outra.

Podemos também dizer que Informação é um processo que visa o conhecimento, ou, mais simplesmente, Informação é tudo o que reduz a incerteza, esse conhecimento em si tem de ter uma compreensão mais profunda para desenvolver nesse sentido práticas mais eficientes de gestão (Silva et al., 2019). Um instrumento de compreensão do mundo e da ação sobre ele (Zorrinho, 1995). E dentro de uma prática social, por meio do estudo é possível analisar vantagens competitivas nas organizações e o impacto da governança de tecnologia da informação como prática para criação e manutenção de valores (Ogécime; Silva, 2019). 
Os investimentos em TI crescem a cada ano e por consequiência, a exigência com relação aos retornos dessa área também tem aumentado gradativamente. Para Gajendran; Brewer, (2012); Woida, (2020) e Arrivabene et al., (2021) as TI podem ser utilizadas para o melhor desempenho organizacional, quando os envolvidos nos processos estão alinhados por meio da cultura organizacional.

A gestão de Tecnologia de Informação e a sua inserção na estratégia empresarial vem a ser na atualidade um fator chave na criação de valor acrescentado e das vantagens competitivas para a empresa. Se, por um lado, ajudam a detectar novas oportunidades e criar vantagens competitivas, por outro, ajudam a defendê-la de ameaças provenientes da concorrência, (Silva; Araújo; Brito, 2018; Ferreira; Brito, 2020).

Conforme os autores Hinton e Heinemann, (2006) mencionam, a gestão da tecnologia da informação é um processo consciente do gerenciamento e administração dos dados gerados por uma empresa, usado nas tomadas de decisões em toda organização. Em todos os níveis organizacionais.

O uso da informação e da tecnologia da informação é distribuído por toda organização. Todas as funções do negócio têm uma medida para atividades, (Hinton; Heinemann, 2006; Nishioka; Przeybilovicz \& Cunha 2014). A análise cultural investiga os conflitos entre os valores defendidos e crenças reais dos membros para entender a cultura e os valores defendidos para a implementação da TI. Entender neste sentido a cultura e os valores defendidos vem a ser a chave para o sucesso nas organizações no curto, médio e longo prazo.

A comparação das situações contextualizadas pela natureza comum das crenças nos projetos dos membros da organização, amplamente discutidos reforçam os "valores defendidos", fornecendo uma base para identificar as questões que levam a níveis bons para implementação das TI.

Perante essa temática contextualizada, emerge a problemática do estudo: qual a importância do alinhamento estratégico da governança corporativa e da governança de tecnologia da informação nas organizações?

O estudo teve como objetivo enfatizar o framework (estrutura) praticado e sua importância no contexto das vantagens competitivas nas organizações. Para atender à crescente demanda por inovação, criatividade, qualidade, melhoria de processos, competitividade dentre tantas outras necessidades neste mundo globalizado. A Criação e manutenção de valores são fundamentais para dar suporte a área ou negócio.

Justifica-se o estudo pois por meio dele pode-se obter informações as quais atendam à crescente demanda por inovação, criatividade, qualidade, melhoria de processos, competitividade dentre tantas outras necessidades neste mundo globalizado real ou virtual. Para Zattar e Marteleto, (2019) o conceito de "Governança", de uma forma geral, remete ao modo como o poder é exercido na administração dos recursos sociais e econômicos de um país.

Por influência da história da implementação da Governança da Tecnologia da Informação (GTI) nas organizações e na representação que os técnicos fazem dela, estão ausentes mecanismos relacionados à estrutura organizacional, e pouco presentes mecanismos de relacionamento com as áreas de negócio (Nishioka; Przeybilovicz \& Cunha, 2014). Indo ao encontro de estudos efetuado por Silva; Marques, (2018) o qual menciona referente a normatização do princípio da neutralidade de rede e o disciplinamento da prática do zero-rating compõem uma das controvérsias mais atuais na área da Governança de TI, área também direcionada a Internet e integra a agenda regulatória de diversos países, dentre os quais se destacam: Canadá, Chile, Holanda e Índia, além da União Europeia, (Silva \& Marques, 2018).

É importante atingir e manter o nível de maturidade de governança de TI compatível com as necessidades dos processos de negócio. Deixando assim evidenciado um gap de pesquisa nesse contexto.

O artigo está estruturado da seguinte forma: Referencial Teórico o qual aborda: Tecnologia da Informação, Governança Corporativa e Governança de Tecnologia da Informação; Vantagem Competitiva nas Organizações; Alinhamento estratégico. Na sequência tem-se a Metodologia; Resultados e Discussão, Conclusões e Recomendações e as Referências. 


\section{Fundamentação Teórica}

\subsection{Tecnologia da informação}

Um termo que engloba todas as formas de tecnologia utilizadas para criar, armazenar, trocar e usar informação em suas várias formas (dados, voz, imagens estáticas e em movimento). Para Porter, (1999), a TI permeia todos os pontos da cadeia de valor das empresas, suportando as atividades das mesmas bem como os elos entre elas.

Os maiores benefícios aparecem quando as estratégias organizacionais, as estruturas e os processos são alterados conjuntamente com os investimentos em TI. Segundo Venkatraman, referido por O'Brien (1996), o verdadeiro poder das TI está em "reestruturar as relações nas redes empresariais para aproveitar um leque mais vasto de competências.

A Tecnologia da Informação (TI) tem um papel relevante na implementação das estratégias empresariais, pois informações confiáveis e íntegras devem estar disponíveis para todos os processos de negócio. As TI permitem assim, ultrapassar todo um conjunto de barreiras na medida em que existe uma nova maneira de pensar, pois em tempo real é possível às empresas agir e reagir rapidamente aos clientes, mercados e concorrência.

Para Lévy (2000), é o uso intensivo das tecnologias que caracteriza a nossa condição humana. Ele rejeita a metáfora do "impacto" (neste caso, negativo), como se o homem fosse um alvo fixo e as tecnologias projéteis externos (Lévy, 2000). As tecnologias são recursos que auxiliam as pessoas a viverem melhor em sociedade, levando em conta o espaço e o tempo.

As tecnologias são necessidades equilibradas pelas necessidades comerciais acordadas (Imbook, 2004). Conhecer novas tecnologias da informação aplicadas aos sistemas de informação torna-se imprescindível. Desta forma, a eficiência com a qual a empresa mobiliza seu conhecimento no desenvolvimento de novos produtos (Ahuja, 2000), sua abordagem em relação ao aprendizado (Sommer; Loch \& Dong, 2009), e sua capacidade de gerar novas ideias (Tiwana, 2008) podem determinar o sucesso ou fracasso de empreendimentos. Tecnologia da Informação (TI) pode ser conceituada como Recursos computacionais (hardware, software e serviços relacionados) que são decorrentes de serviços de comunicação, processamento e armazenamento de dados.

\subsection{Governança corporativa e governança de tecnologia da informação}

Governança em TI na governança corporativa é a peça chave para o alinhamento entre as diretrizes e estratégias da organização nas ações de TI (Severiano Junior \& Cunha, 2018). A Governança Corporativa tornou-se familiar para os executivos das grandes empresas a muito tempo. Ela trata dos caminhos pelos quais os acionistas das corporações asseguram que terão retorno de seus investimentos (Shleifer \& Vishny, 1997). O termo Governança Corporativa ou o movimento pela Governança Corporativa passa a despontar no cenário mundial a partir do início da década de 80 nos Estados Unidos.

Não só as grandes empresas, mas as pequenas e médias empresas entenderam a importância de investir nas melhores práticas no intuito de alcançar uma gestão mais eficaz e a maior transparência agregando valor e longevidade nos negócios (Severiano Junior \& Cunha, 2018). No Brasil as práticas de Governança Corporativa passam pela formação dos conselhos de administração, os quais tiveram origem com as multinacionais norte-americanas, como forma de seguir os princípios e modelo de gestão da matriz.

Demonstrar quantidade e qualidade das informações ao mercado passou a ser um diferencial para empresas de capital aberto (Migue Peixe, et al., 2018; Lopes; Beuren \& Vicente, 2021). Para Jesus \& Dalangaro, (2018) o resultado econômico das ações realizadas na gestão pode elevar a governança a uma maior representatividade na sociedade, além de mensurar a eficiência na produção da transparência e da accountability.

Mais recentemente, a expressão passou também a ser adotada em tecnologia da informação, para se referir aos critérios de definição, gestão e acompanhamento de resultados dos investimentos em TI (Weill, 2004). Governança de TI faz parte da Governança da Empresa, já a Governança Corporativa foca em como os financiadores se asseguram de que os 
gestores vão dar retorno de seus investimentos. No Quadro 1 Haes et al., (2004) menciona diferenças entre Governança Corporativa e Governança de TI:

Quadro 1 - Governança Corporativa e Governança de TI.

\begin{tabular}{|c|c|}
\hline GOVERNANÇA CORPORATIVA & GOVERNANÇA DE TI \\
\hline $\begin{array}{c}\text { Financiadores se asseguram de que os } \\
\text { gestores vão dar retorno de seus investimentos. }\end{array}$ & $\begin{array}{l}\text { Diretoria assegura que o CIO e o } \\
\text { arcabouço de TI irão aditar valor para a } \\
\text { organização. }\end{array}$ \\
\hline $\begin{array}{c}\text { Financiadores se asseguram de que os } \\
\text { gestores não vão desapropriar o capital que } \\
\text { investiram ou investir em ideações maldosa. }\end{array}$ & $\begin{array}{c}\text { Diretora assegura de que o CIO e o } \\
\text { arcabouço de TI não irão desapropriar o capital } \\
\text { investido ou investir em ideações ruins. }\end{array}$ \\
\hline Financiadores controlam os gestores. & \multicolumn{1}{|c|}{ Diretoria controla o CIO e o arcabouço } \\
de TI.
\end{tabular}

Fonte: Haes et al., (2004). Baseado em: Shleifer A. \& Vishny W., (1997), A survey on corporate governance, The Journal of Finance, 52(2)

Ainda complementando o mencionado no Quadro 1, destaca-se que, "Governança" vem da palavra grega "kubernan", que significa navegação ou o processo de continuamente orientar e ajustar. O termo é utilizado em uma grande variedade de disciplinas, incluindo direito, políticas públicas, medicina e, especialmente, na administração.

Entretanto, a popularização do seu uso, necessariamente, não tornou mais preciso o seu significado, especialmente em decorrência da aplicação multidisciplinar (Lunardi, 2008). Para Assis (2011) a governança de TI concentra-se no desempenho e transformações para atender as demandas futuras do negócio ao longo do tempo, trabalhando praticamente com orientações de negócios externamente. Sendo a Tecnologia da informação um recurso estratégico para as empresas a governança da tecnologia da informação serve para orientar e fazer estes ajustes no uso da tecnologia da informação, nas empresas.

Preocupa-se com a definição de papéis, responsabilidades, processos, políticas, padrões, diretrizes para o uso adequado dos recursos da TI, planejamento estratégico da TI e projetos relevantes para sua implantação e sustentação, além do controle de investimentos e orçamento, (Assis, 2011). Isto evidencia que há espaço para que a organização amplie sua capacidade processual, agregando práticas reconhecidas.

A alavancagem tecnológica que o mundo presenciou no século XXI, é de extrema importância para as empresas estarem sempre atualizadas quanto as melhores soluções de sistemas que facilitem as rotinas dos departamentos, que tragam informações fidedignas e que ainda façam previsões e projeções de acordo com os históricos de movimentações da empresa, tudo isso com foco em responsividade, qualidade da informação, segurança, disponibilidade e capacidade.

Esta alavancagem tecnológica consiste em um cenário onde a tecnologia modifica o papel e o perfil dos colaboradores e departamentos, pois com a melhoria e expansão dos sistemas o gestor tem como observar aspectos mais estratégicos da empresa, olhar para frente e não apenas ficar conciliando os acontecimentos, buscando falhas e desvios das movimentações já ocorridas.

As empresas competem cada vez mais para obter os resultados rápidos, com clareza, mais próximos da realidade e com a maior assertividade. Buscar soluções fundamentadas em Tecnologia da Informação no mercado tornou-se um diferencial para as empresas e é essa ação que pode fazer uma empresa superar a concorrência, porém a dificuldade é se manter corretamente. 
A tecnologia da informação é uma das engrenagens que fazem com que as empresas se movimentem, e para isto a tecnologia da informação deve sempre ser tratada com seriedade e prioridade, pois também é considerada um fator diferencial nos sucessos das empresas que a utilizam.

Para que isso ocorra a organização deve estar com uma boa estrutura formulada de GC e GTI. Na Figura 1 tem-se estrutura (framework) de governança corporativa voltada para a área de tecnologia da informação e para melhor compreensão.

Figura 1 - Governança Corporativa.

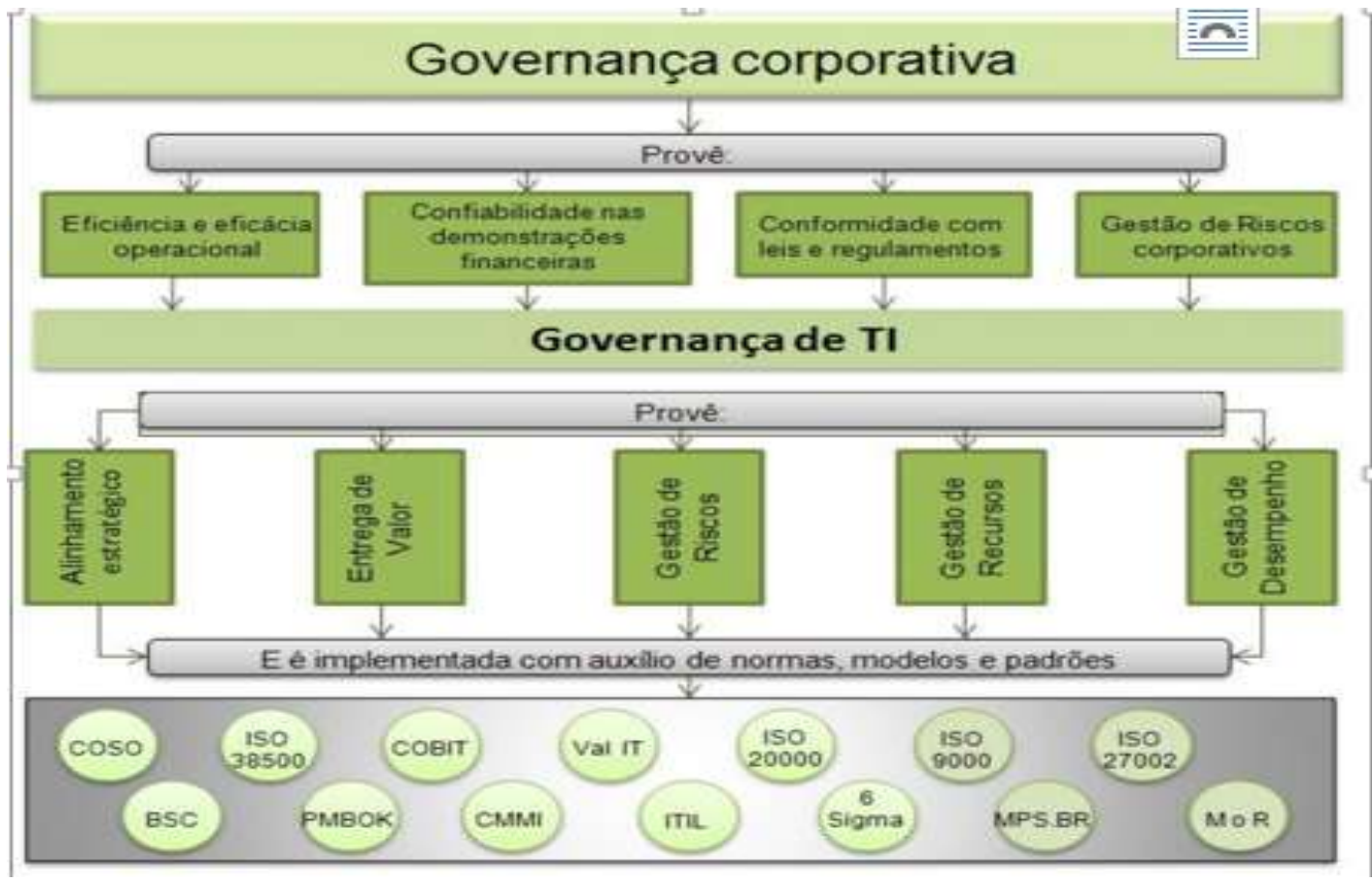

Fonte: Imagem adaptada de: Feely, Dan. Getting Governance Right. Info Source, v. VII, n. 3.

O framework ilustrado na Figura 1, exemplificado na primeira camada por um conjunto de normas, modelos, padrões (ITIL, ISO, ETC) que são empregados na operacionalização, gestão tática e estratégica de serviços de Tecnologia da Informação, bem como na melhoria contínua dos mesmos.

Está camada está associada a Governança de TI, onde busca-se o alinhamento estratégico, a entrega de valor, a gestão de riscos, recursos e desempenho. Estes, quanto mais delineados, compreendidos pelos envolvidos, em suas estratégias, objetivos, ações e metas, mais assertivos serão os resultados alcançados.

A governança corporativa, quando relacionada ao ativo de Tecnologia da Informação, em suas estratégias, tem como propósito medir o desempenho da TI e sempre manter o alinhamento entre tecnologia e negócios, buscando sempre eficiência e eficácia, confiabilidade, conformidade, gestão de riscos e resultados com previsibilidade e transparência.

A partir do momento que os processos organizacionais começaram a ser assistidos por tecnologias da informação, muitos acadêmicos e profissionais preocuparam-se em estudar as relações entre causas e efeitos do uso da TI para a realização dos objetivos organizacionais (Van Gremberger, 2004). Essas pesquisas foram importantes e contribuíram com seu framework, para uma variedade de definições de governança de TI que encontramos atualmente na literatura (Almeida; Souza, 2019).

Este framework de Governança de TI tem sido operacionalizado no mundo todo, em conjunto com estas práticas, de onde devem ser extraídos os pontos que atinjam os objetivos do programa de Governança.

O termo Governança Corporativa ou o movimento pela Governança Corporativa passa a despontar no cenário mundial a partir do início da década de 80 nos Estados Unidos. No Brasil surgiu em 1999, elaborado pelo Instituto Brasileiro de 
Governança Corporativa (IBGC) com o apoio da Bolsa de Valores de São Paulo: Brasil Bolsa Balcão - (B3), (Almeida; Souza, 2019).

$\mathrm{Na}$ investigação dos fenômenos complexos de administração e sistemas de informação (SI), exigem-se, por vezes, abordagens que caracterizem a ação humana. A Teoria da Representação Social (TRS) é uma alternativa ao desenvolvimento de estudos focados não apenas em fatos, dados e opiniões formais, mas também no entendimento das percepções e valores compartilhados pelos sujeitos (Vergara \& Ferreira, 2005; Nishioka; Przeybilovicz \& Cunha 2014; Batel \& Castro, 2018; Augoustinos, 2019).

A TRS tem em sua origem o estudo feito por Serge Moscovici (1978), quando pesquisou na população parisiense o entendimento sobre a psicanálise, um conceito que se popularizava na época. Na Figura 2 o autor cita estas abordagens que caracterizem a ação humana por meio da Governança de TI e utilizando a Teoria da Representação Social (TRS).

Figura 2 - Governança de TI utilizando TRS.

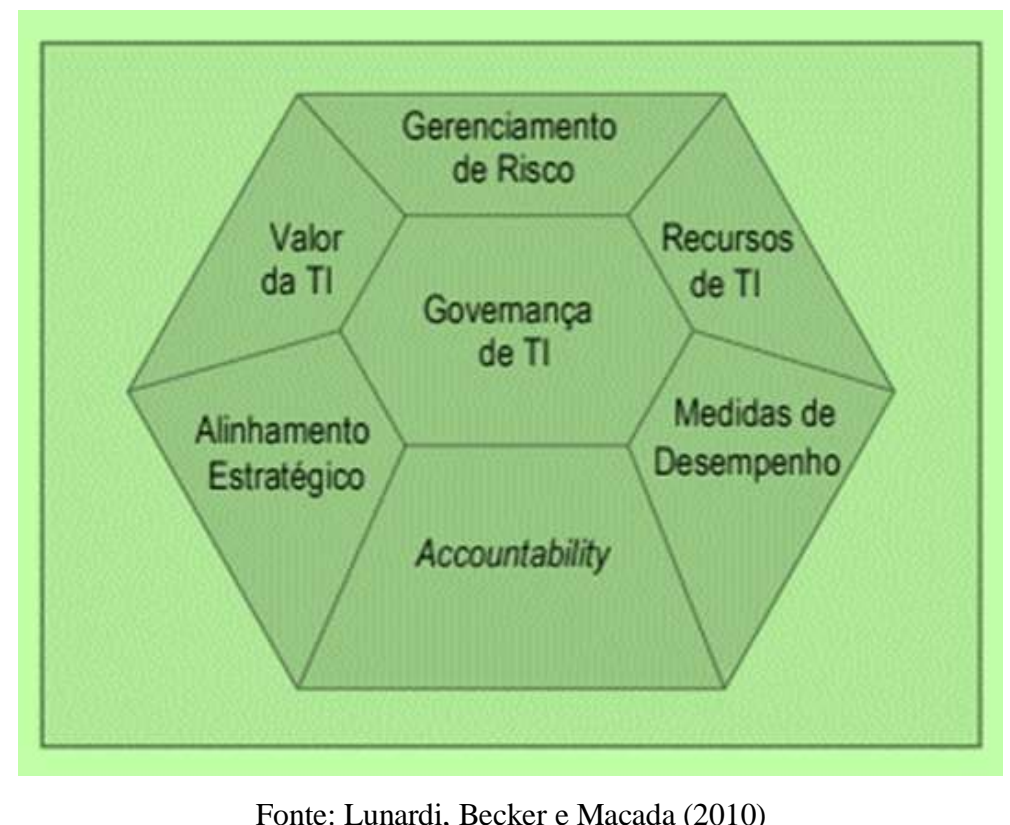

O objetivo central do Código das Melhores Práticas da Governança Corporativa é indicar caminhos para todos os tipos de empresas (sociedades por ações de capital aberto ou fechado, limitadas ou sociedades civis), visando melhorar seu desempenho e facilitar o acesso ao capital (Steinberg, 2003). A implementação efetiva da Governança de TI é possível com o desenvolvimento de um framework (estrutura) organizacional específico fundamentado em COBIT e ITIL.

Em estudos efetuados por autores como Sandonato, (2007); Severiano Junior \& Cunha, (2018) ITIL e o COBIT não estão em desacordo, mas são complementares, pois o ITIL visa melhorar as áreas de TI, COBIT, do ponto de vista da governança corporativa (Santos; Paula, 2016). COBIT é uma matriz que unifica mundialmente todos os padrões de tecnologia da informação, que sofre influência de alguns modelos como: ITIL, CMMI e ISO17799, (Sandonato, 2007; Severiano Junior \& Cunha, 2018). São objetivos do COBIT: a valorização da empresa o aumento do fluxo de investimento e a proteção dos clientes, diretores, acionistas e funcionários da empresa.

$\mathrm{O}$ conceito de COBIT não se trata de uma simples metodologia. Trata-se de uma ferramenta robusta de gerenciamento de todo o ambiente computacional da organização, (Severiano Junior \& Cunha, 2018).

A Sigla COBIT que se origina do termo em inglês Control Objetives for Information and related Technology, ou se traduzido em português Objetivos de Controles (diretrizes) para Informações relacionadas a Tecnologias. Em âmbito mundial, 
trata-se do modelo para governança de tecnologia da informação mais adotado mundialmente, principalmente devido sua amplitude e robustez em relação aos demais modelos de trabalho (Severiano Junior \& Cunha, 2018).

Segundo Selig, (2016); Fernandes, et.al., (2018), em seus estudos em empresas do segmento de TI "na descrição do COBIT e do ITIL é possível observar que os processos conseguem alinhar o ciclo de vida da preparação até a entrega da solução do cliente, porém, não especifica quais as ferramentas de sistemas e dispositivos de comunicação que devem ser utilizados como suporte à implementação de toda a solução. Pode-se dizer que essas ferramentas e dispositivos são soluções de mercado disponíveis comercialmente (Severiano Junior \& Cunha, 2018; Fernandes, et.al., 2018).

ITIL e o COBIT são complementares, pois o ITIL visa melhorar as áreas de TI do COBIT, do ponto de vista da governança corporativa em um âmbito geral, sendo assim o COBIT acaba suportando a empresa com mais eficácia, com a adoção de seus processos no ambiente de TI, (Fernandes, et.al., 2018). Já o autor Selig, (2016), menciona que enquanto o objetivo de um framework é gerar maior valor competitivo para o negócio, o COBIT especificamente se preocupa que a TI seja gerida de forma holística e integrada, possibilite a realização de benefícios, a melhoria dos níveis de risco e a otimização do uso dos recursos de TI, (Selig, 2016).

O COBIT é, portanto, um guia para a gestão de TI, utilizado como um modelo para gestão que abrange o nível estratégico, tático e uma parte do operacional. Por sua vez, o Information Technology Infrastructure Library (ITIL) abrange o nível operacional e uma parte do tático (Santos; Paula, 2016).

No contexto da Governança Corporativa, a tecnologia da informação tem seu destaque, em função da evolução do processo de gestão e operacionalização. O poder de decisão tem se fundamentado cada vez mais na competitividade gerada pelo princípio da oportunidade, está fundamentada nos princípios de governança. O guia DAMA-DMBOK (2012) corrobora que "nenhuma organização pode ser eficaz sem dados de alta qualidade". Estudos efetuados por Nishioka; Przeybilovicz \& Cunha, (2014), evidenciam que a representação da Gestão de Tecnologia de Informação dos profissionais de uma determinada empresa a qual os autores supracitados chamaram de "Alfa", altamente competitiva operacionalmente, estava ancorada no conjunto de regras do ITIL, com foco em controle e uma visão interna à área de TI.

A Information Technology Infrastructure Library ou Biblioteca de Infraestrutura de Tecnologia da Informação (ITIL) é considerada como a mais reconhecida referência de práticas de gerenciamento de serviços de TI do mundo. No guia DamaDmbok (2012), guia para o corpo de conhecimento em gerenciamento de dados encontra-se a seguinte menção, cada organização precisa gerenciar eficazmente os seus importantes dados e recursos de informação.

Fundamentado nestas referências, conforme supracitado acima, empresas que adotam o COBIT e o ITIL, apresentam atributos competitivos que diferenciam as empresas em seus modelos de negócio junto a seus clientes internos e externos.

\subsection{Vantagem competitiva nas organizações}

Vantagem competitiva nas organizações é a capacidade de uma organização se diferenciar de seus concorrentes. No ambiente organizacional a relação entre as pessoas e as organizações devem ser desenvolvidas e mantidas, visando o fortalecimento das organizações, este relacionamento proporciona um melhor atendimento as demandas e consequentemente a manutenção da vantagem competitiva almejada.

A cultura, classe social, família, amigos, instituições, experiências e leituras, valores, crenças, atitudes, emoções, competências e motivação são fatores que influenciam no comportamento humano e devem ser levados em consideração para o processo de agregação de valor nas organizações competitivas.

A satisfação dos clientes é alcançada a partir de diversas ações que as empresas precisam executar, assim, oferecer produtos e serviços de qualidade, além de preços e prazos, estes são alguns pontos que podem influenciar na satisfação do cliente e na competitividade das organizações. 
No contexto atual a vantagem competitiva nas organizações está relacionada aos clientes, eles possuem o direito de escolha associados a qualidade do serviço e aos produtos ofertados. Se uma empresa não procurar conhecer seus clientes para atender suas necessidades e expectativas, certamente haverá um concorrente para fazê-lo.

Nesse sentido, acentuado com tanta inovação por meio das tecnologias nas organizações, o uso da governança corporativa e de governança de tecnologia da informação tem fator decisivo na vantagem competitiva, empoderando no mercado globalizado e resultando em satisfação do cliente. No quadro 2 menciona-se os cinco passos para criar a vantagem competitiva nas organizações.

Quadro 2 - Cinco passos para criar a Vantagem Competitiva.

\begin{tabular}{|l|l|l|l|l|}
\hline \multicolumn{1}{|c|}{ (I) } & \multicolumn{1}{|c|}{ (II) } \\
$\begin{array}{l}\text { Invista na qualidade do } \\
\text { produto }\end{array}$ & $\begin{array}{l}\text { Assistência } \\
\text { diferenciada }\end{array}$ & $\begin{array}{l}\text { (III) } \\
\text { Equipe motivada e satisfeita é } \\
\text { um grande diferencial }\end{array}$ & $\begin{array}{c}\text { (IV) } \\
\text { Seja inovador }\end{array}$ & $\begin{array}{l}\text { Bom relacionamento } \\
\text { com cliente }\end{array}$ \\
\hline
\end{tabular}

Fonte: Autores (2019) adaptado de (Porter, 1989).

A abordagem da vantagem competitividade tem papel central em estudos de empresas ou de arranjos produtivos locais, pois, assim como a inovação, representam fatores de sustentação das atividades em qualquer sistema de produção (Porter, 1989).

\subsection{Alinhamento estratégico}

O alinhamento estratégico é obtido por meio de boa comunicação e transparência da organização. Estar alinhado exige que as organizações alterem sua dependência em um único caminho, buscando refletir, planejar, desenvolver capacidades estratégicas para com as pessoas, utilizando e delineando os recursos de TI que gerem ações efetivas para o negócio (Schmitt; Raisch \& Volberda, 2018).

A noção de alinhamento estratégico origina-se da principal proposição na qual o desempenho organizacional é uma consequência da estratégia de negócios, (Ilmudeen; Bao \& Alharbi, 2019). No atual ambiente de negócios hiper-competitivo, alinhar a estratégia de TI à estratégia de negócios tornou-se um problema crucial e continua a ser uma preocupação dos executivos de negócios e TI (Wu et al., 2015; Luftman et al., 2017).

Embora o alinhamento tenha sido estudado de forma ampla, os estudiosos usaram várias conceitos e definições para o alinhamento (Gerow et al., 2016). O "alinhamento" como o elo entre as estratégias de TI e de negócios, enquanto outros definem como a extensão com que o planejamento de SI apoia o planejamento de negócios. Conforme Ilmudeen; Bao \& Alharbi, (2019) o alinhamento é um construto multidimensional e as empresas tradicionalmente encontram obstáculos para alcançá-lo.

A TI e sua relação investimento-empresa-desempenho deve focar não apenas no alinhamento dos processos, mas também nas dimensões de alinhamento estratégico. Mediante a estudos realizados pelos autores Ilmudeen; Bao e Alharbi (2019) o alinhamento estratégico de TI pode ser ampliado para três dimensões. (1) estratégia de TI, (2) estratégia de negócios, (3) destacando a complementaridade entre a estratégia de TI e estratégia de negócios. Assim, os estudiosos precisam especificar as dimensões de alinhamento e desempenho da empresa para entender as nuances padrão dos construtos alinhamento-desempenho (Gerow et al., 2016).

Os investimentos em TI não garantem melhorar o desempenho organizacional (Peng et al., 2016). A avaliação do mapeamento estratégico de TI com a estratégia de negócios, em relação aos investimentos em TI, é mais significativa se traduzido em ações planejadas, equacionadas e mensuradas objetivando as entregas de benefícios propostas no negócio. A 
direção estratégica inovadora permite que a empresa supere os concorrentes, mantendo-a de forma inovadora e reforçando a competitividade da empresa em sua posição no mercado (Theodosiou et al., 2012).

\section{Metodologia}

O estudo possui como delineamento metodológico características de pesquisa bibliográfica, exploratória, documental, descritiva, explicativa e qualitativa.

Buscando nesse sentido fornecer uma visão geral do problema, do tipo aproximativa pela compreensão do investigador em relação ao fenômeno ou caso que resulta no aprimoramento de ideias (GIL, 2017). O aprimoramento dessas ideias pode ser efetuado por meio de uma boa governança a qual reflete em serviços de qualidade, resultados e maior transparência para os envolvidos, se apropriando da utilização dos recursos de tecnologia da informação como vantagem competitiva nas organizações.

A forma de coletar os dados possui natureza instrumental e deve viabilizar as informações objetivando a análise e a explicação dos aspectos em estudo. Coletar dados é uma observação da vida real que, quanto a sua natureza, pode ser dividida da seguinte forma (MICHEL, 2009): (I) Dados Primários: extraídos diretamente das fontes pesquisadas, tais como as pesquisas realizadas em campo. (II) Dados Secundários: extraídos por meio de análise documental, como relatórios, livros, jornais, revistas e websites.

Na pesquisa fez-se uso dos dois tipos de dados. Para efetivação de uma pesquisa torna-se fundamental realizar algumas etapas cientificas, Cooper \& Schindler (2016), corroboram que num contexto amplo de etapas tem-se: (I) descobrir os problemas de pesquisa; (II) escolher um problema no qual se concentrar; (III) elaborar o planejamento de pesquisa; (IV) escolher os métodos de coleta de dados; (V) analisar, interpretar e produzir relatórios sobre o objeto em estudo; e (VI) tomar a decisão gerencial de como resolver o problema.

Nesse contexto para a pesquisa efetivar-se teve-se como intuito enfatizar o framework praticado e sua importância na conjuntura das vantagens competitivas nas organizações. Resultando assim no atendimento à crescente demanda por inovação, criatividade, qualidade, melhoria de processos, competitividade dentre tantas outras necessidades neste mundo globalizado real ou virtual.

\section{Resultados e Discussão}

Ao delinear o estudo referente o tema Governança Corporativa e Governança de Tecnologia da Informação como Vantagem Competitiva nas Organizações, torna-se factível a evidenciação em relação a questão crítica referente aos termos de gerenciamento. O desafio é encontrar as conexões gerenciais entre a tecnologia da informação, as soluções propostas e as ideais aplicáveis, que atendam às necessidades de negócios, e os objetivos do mesmo.

Resultados organizacionais geralmente são articulados com metas e indicadores de desempenho incorporando-se a um plano estratégico. Esta lógica se manifesta ao trabalhar as técnicas de planejamento, visando estratégias, objetivos, ações, metas, indicadores e recursos. Busca-se claramente atingir a satisfação do usuário e os objetivos organizacionais por meio da entrega de benefícios e otimização de recursos, estes nem sempre são atingidos, tendo em vista como esse processo é operacionalizado, gerenciado, documentado.

O estado da arte, são os resultados das organizações que na atualidade são impulsionados por estratégias e sua operacionalização por meio das tecnologias de informação. Há uma enorme onda de interesse em ferramentas tecnológicas de gestão e técnicas, que ajudem a incorporar informações e tecnologias na formulação de estratégias de negócios, visando investimentos com base em necessidades reais de negócios e seus stakeholders.

A Governança Corporativa alinhada com a Governança de Tecnologia da Informação, gera uma sinergia positiva 
entre o CEO (Chief Executive Officer), que representa o diretor executivo de uma empresa e todos seus anseios, com o CIO (Chief Information Officer), responsável pela gestão da área de Tecnologia de Informação. Neste contexto, justifica-se e muito em função da utilização vertiginosa da tecnologia de informação, seus resultados, a digitalização do negócio, segurança, compliance, Lei Geral de Proteção de Dados - (LGPD), entre outros. Este, responsável por toda a informática de uma empresa, deve, juntamente com sua equipe, estar alinhado com às tecnologias emergentes e as melhores práticas do mercado, sem se descuidar da estrutura de Tecnologia da Informação, vanguarda para impulsionar as melhores estratégias que possam vir a surgir das mentes brilhantes no contexto organizacional.

A Tecnologia da Informação cada vez mais tem evidenciado a sua importância pois é um recurso estratégico dentro das empresas, auxiliando no monitoramento de processos tecnológicos, estes relacionados ao tratamento dos dados. A Governança Corporativa é atuante na formulação de estratégias para orientar as empresas na avaliação das incertezas os financiadores controlam os gestores. E na Governança de Tecnologia da Informação a diretoria controla o CIO.

Neste objeto vem a ser o "CIO", responsável por toda a informática de uma empresa, precisando estar antenado às tecnologias da atualidade, gerenciar os recursos digitais, sistemas internos e externos e implementar sistemas com inteligência para alcançar os objetivos da empresa e a estrutura de Tecnologia da Informação, assim todo este contexto bem alinhado por meio de estratégias obter-se-á uma excelente vantagem competitiva nas organizações e mercado. Nas empresas encontram-se pessoas mais e menos competitivas. Por meio da percepção, da compreensão, do aprendizado e da adaptação aos ambientes organizacionais, pode-se buscar melhoria nas tomadas decisórias, no desempenho das pessoas, no conhecimento e na previsão do mundo que nos cerca, alavancando a competitividade das empresas.

\section{Conclusão e Recomendações}

Desenvolver estratégias de negócios é fundamental, mesmo sendo estas estratégias de negócios facilitadas pela tecnologia da informação e suas oportunidades. Porém a dificuldade está no espaço e operacionalização entre elas, a estratégia e a Tecnologia da Informação.

Todos os atores de uma organização devem combinar seus esforços nesta tarefa difícil, se quisermos realmente melhorar o desempenho dos negócios seja por meio da implantação e/ou melhoria de sistemas de informação, infraestrutura, governança e demais tecnologias eficazes. Dois fatores que se leva em consideração no desempenho do negócio vem a ser: (i) comunicação e (II) gerenciamento.

Quando se pondera a grande diversidade de habilidades necessárias para adquirir e implantar tecnologias da informação, a comunicação entre as diferentes partes torna-se difícil e necessária, e os gerentes muitas vezes parecem incapazes de lidar com os problemas que surgem.

A janela de vantagem (período de tempo que leva para os concorrentes alcançar e igualar a vantagem estratégica) tornou-se questão crítica em termos de gerenciamento. Esta ficou mais curta evoluída em segundos, horas, dias, meses, não mais anos - até que a capacidade de gerenciar a mudança se tornou a mais importante.

Existe uma cultura de "atirar coisas para o outro lado do muro", admitindo que os dois mundos nunca se encontrarão. A globalização, suas relações comerciais e influências são provas contrárias disso. Neste contexto, a tecnologia da informação tem se tornado um diferencial, direcionando e impulsionando resultados, fundamentados em custo/benefício e oportunidades.

Competências e habilidades em gerenciar negócios e estratégias dinâmicas, muitas vezes com resultados imediatos, requer dos profissionais atuais, um perfil arrojado para influenciar, gerar oportunidades, gerenciar mudanças e potencializar retornos sob investimentos. 
Especialistas qualificados nos negócios e necessidades da organização (gerenciar estratégias, recursos e benefícios) atingem melhores resultados por meio da governança corporativa e da governança de TI. Porém o que é predominante nas empresas de modo geral, é que ainda existe uma lacuna entre a Pessoa de "negócios" e a Pessoa de "TI".

O mundo empresarial vê a tecnologia da informação como um fardo e um custo, que tem que ser contido e administrado. A maneira de resolver essa diferença de atitude é entender a interseção dos dois mundos.

A realidade é que não se deve tratar como conexões entre esses dois mundos e sim a necessidade de compreendê-los visando estratégias, objetivos, ações, recursos e resultados que a tenham sucesso e consequentemente os negócios. As organizações que adotam um framework de boas práticas, se delineando aos demais setores, consequentemente tem resultados mais assertivos e estes evidenciam o alinhamento.

A Governança Corporativa e a Governança de TI devem estar atentas a obtenção de benefícios mensuráveis e oportunos em relação ao investimento em TI, se estão sendo implementados de maneira correta, completa e eficaz, se estão bem gerenciados, e se está conduzindo sua estratégia de TI ou ela está impulsionando. Existe uma visão clara de um futuro melhorado, onde as estratégias de TI e de negócios estão alinhadas.

Há também uma preocupação generalizada sobre a falta de capacidade de TI em organizações de todos os tipos. Alguns críticos associam esta falta de competências relacionadas a gestão do negócio e da TI. O desafio é encontrar as conexões gerenciais entre a TI, as soluções propostas e necessárias de tecnologia de informação, que atendam às necessidades de negócios, e os objetivos o negócio.

Sugere-se para trabalhos futuros investigar dois fatores importantes em consideração ao desempenho do negócio: (i) comunicação e (II) gerenciamento de indicadores; observar se as organizações têm se preocupado com estes propósitos. Contemporaneamente servem para a performance de qualquer negócio. Nos estudos percebe-se uma lacuna entre a Pessoa de "negócios" e a de "TI", torna-se explicito a necessidade de amenizar esta lacuna entre as respectivas partes, objetivando o melhoramento da vantagem competitiva nas organizações.

\section{Referências}

Almeida, R. S. \& Souza, W. A. (2019) Implementação dos mecanismos de governança de tecnologia da informação em uma universidade pública. Navus. Revista de Gestão e Tecnologia. 9(3), 136-149.

Agrawal, A., Gans, J. \& Goldfarb, A. (2018). How AI will Change Strategy: A Thought Experiment. Harvard Business Review. https://hbr.org/2017/10/howai-will-change-strategy-a-thought-experiment/Accessed February 20.

Augoustinos, M. (2019). Dialoguing the difference: A reply to Batel and Castro's 'Re-opening the dialogue between the Theory of Social Representations and Discursive Psychology'. British Journal of Social Psychology, 58, 423-427.

Ahuja, G. (2000). Collaboration Networks, Structural Holes, and Innovation: A Longitudinal Study. Administrative Science Quarterly, $45(3), 425$. $10.2307 / 2667105$.

Arrivabene, A., Sassi, R. J., Andrelo, P. F. A. \& Moura, M. L. A. de O. (2021) Analysis of the Impact of Adequacy on Operational Information Technology Processes to the Requirements of the Sarbanes - Oxley Act in a Iinancial Company. Research, Society and Development, 10(1), e 7710111374, 2021(CC BY 4.0) | http://dx.doi.org/10.33448/rsd-v10i1.11374.

Assis, C. B. (2011). Governança e gestão da tecnologia da informação: diferenças na aplicação em empresas brasileiras / C.B. Assis.

Bhagwati, J., Panagariya, A. \& Srinivasan, T. N. (2004) “The Muddles over Outsourcing,” Journal of Economic Perspectives, 18(4), 93-114.

Batel, S. \& Castro, P. (2018). Re-eningthedialoguebetweentheTheoryofSocialRepresentations and Discursive Psychology for examining the construction and transformation of meaning in discourse and communication. British Journal of Social Psychology, v.57, p.732-753. https://doi. org/10.1111/bjso.12259.

Cooper, D. R. \& Schindler, P. S. (2016). Métodos de pesquisa em Administração. (12a ed.), Bookman.

Couto, F. \& Ishitani, L. (2018). Implementation of ITIL Configuration Management module: a case study (Implantação do modulo de Gestão de Configuração do framework ITIL: um estudo de caso). iSys: Revista Brasileira de Sistemas de Informação (Brazilian Journal of Information Systems), 11(3), 70-89.

Ferreira, D. O., \& Brito, M. L. A. (2020). Qualidade do atendimento no comércio de confecções: Um estudo na cidade de Currais Novos-RN. Research, Society and Development, 9, 93922093. 
Feely, D. (2007) Getting Governance Right. Info Source, v. VII, n. 3. http://www.transforming.com/tsi_news/best_consulting_firm_news letters_december07.html

Gajendran, T., \& Brewer, G. (2012). Cultural consciousness and the effective implementation of information and communication technology. Construction Innovation, 2(12), 179-197.

Gerow, J. E., Grover, V. \& Thatcher, J. (2016). “Alignment's nomological network: theory and evaluation”, Information \& Management, 53(5), 541-553.

Gil, A. C. (2017). Como Elaborar Projetos de Pesquisa. (6a ed.), Atlas.

Dama-Dmbok. (2012). Guia Para o Corpo de Conhecimento em Gerenciamento de Dados.

Fernandes, T. C., Maia; C. I., Salvetti, N., Magalhães, F. L. F. de \& Fernandes, A. A. (2018). Influence of Dev Ops practices in IT management processes according to the COBIT 5 model. ISSN 2237-4558. Navus. 8(1), 20-31.

Haes, S. \& Gremberger, W.V. (2004). IT Governance and Its Mechanisms, Information Systems Control Journal, v. 1.

Hinton, M. \& Heinemann, B. (2006) Introducing Information Management: the business approach, 1 st ed., Burlington: Elsevier Butterworth - Heinemann.

Ilmudeen, A., Bao, Y. \& Alharbi, I. M. (2019). How does business-IT strategic alignment dimension impact on organizational performance measures Conjecture and empirical analysis. Journal of Enterprise Information Management @ Emerald Publishing Limited 1741-0398 10.1108/JEIM-09-2018-0197.

Imbok. (2004). Collected and edited by Andy Bytheway Cape Technikon, Cape Town, South Africa. This version of IMBOK is dated 23 August 2004 p. 1163.

Jesus, M. R. de. \& Dalongaro, R. C. (2018). Governança Corporativa na Administração Municipal: Uma Análise do Resultado Econômico da Secretaria de Fazenda De Ijui (Rs) - Brasil. Revista Observatório de la Economía Latinoamericana, abril 2018. https://www.eumed.net/rev/oel/2018/04/administracaomunicipal-brasil.html.

Lévy, P. (2000). As Tecnologias da Inteligência. O futuro do pensamento na era da informática. Tradução Carlos Irineu da Costa. Editora, 34,

Liang, H., Saraf, N., Hu, Q. \& Xue, Y. (2007) “Assimilation of enterprise systems: the effect of institutional pressures and the mediating role of top management," MIS Quarterly, 31(1), 59-87.

Lopes, I. F., Beuren, I. M. \& Vicente, E. F. R. (2021). Association Between Risks Management Disclosure and Corporate Governance and Performance in Companies with ADRs. Revista Evidenciação Contábil \& Finanças, 9(1), 5-21.

Lunardi, G. L. (2008). Um Estudo Empírico e Analítico do Impacto da Governança de TI no Desempenho Organizacional. Universidade Federal do Rio Grande do Sul. p. 201.

Lunardi, G. L., Becker, J. L. \& Maçada, A. C. G. (2010). Impacto da adoção de mecanismos de governança de TI no desempenho da gestão de TI: uma análise baseada na percepção dos executivos. Revista da Ciência da Administração, 12(28), 11-39.

Luftman, J., Lyytinen, K. \& ZVI, T.B. (2017) "Enhancing the measurement of Information Technology (IT) business alignment and its influence on company performance", Journal of Information Technology, 32(1), 26-46.

Michel, M. H. (2009). Metodologia e Pesquisa Cientifica em Ciências Sociais. (2a ed.), Atlas S.A.

Miguel Peixe, A. M., Korontai, J. N., Higa, N., Kowalski, D., Clemente, A. \& Severo Peixe, B. C. (2018). Qualidade Informacional Versus Nível De Governança Corporativa: Análise de Correlação das Empresas do Setor de Energia Elétrica. Revista de Contabilidade da UFBA, 12 (3), 65-85.

Mithas,S. \& Rust, R.T. (2016). Howinformation technology strategy and investments influence firm performance: conjectures and empirical evidence", MIS Quarterly, 40(1), 223-245.

Nascimento, H. L. \& Brito, M. L. A. (2020). Organizational climate and job satisfaction: a case study in a supermarket. Research, Society and Development, 9, 112921584 .

Nbr/Iso/Iec 17799. (2005). Tecnologia da Informação: Código de prática para a gestão da segurança da informação. Rio de Janeiro. Associação Brasileira de Normas Técnicas.

Nishioka, A. T., Przeybilovicz, E. \& Cunha, M. A. (2014). Governança de tecnologia da informação: uma investigação sobre a representação do conceito -

R.Adm. 49(2), 307-321.

Moscovici, S. (1978). A representação social da psicanálise. Zahar.

Ogécime, M. \& Silva, R. A. da. (2019). From The Levels of Memory to Their Infotechnicist Unfolding in The Information Society. Inf. \& Soc.:Est., João Pessoa, 29(2), 23-42.

O'brien Virgínia. (1996). MBA intensivo em Gestão. Instituto Superior de Gestão. Abril/ Controljomal Editora, Lda.

Peng, J., Quan, J., Zhang, G. \& Dubinsky, A.J. (2016). Mediation effectof business processand supply chain management capabilities on the impact of IT on firm performance: evidence from Chinese firms", International Journal of Information Management, 36(1), 89-96.

Porter, M. E. (1989). A vantagem competitiva das nações. (7a.ed.), Campus.

Theodosiou, M., Kehagias, J. \& Katsikea, E. (2012). Strategic orientations, marketing capabilities and firm performance: an empirical investigation in the context of frontline managers in service organizations", Industrial Marketing Management, 41(7), 1058-1070. 
Santos, D. F. dos. \& Paula, L. M. de. (2016). Alinhando a governança de TI com os negócios: um estudo entre COBIT e ITIL. Revista de Tecnologia Aplicada - RTA. 5(3), 16-26.

Sandonato, S. F. (2019). A Importância dos modelos de trabalhos de controle de processos para gestão efetiva da tecnologia da informação, 2007. http//www.abepro.org.br/biblioteca/ENEGEP2007_TR630468_0476.pdf.

Selig, G. J. (2016). IT Governance - an integrated framework and roadmap: how to plan, deploy and sustain for improved effectiveness. Journal of International Technology and Information Management. 25(1), 55-76.

Severiano Junior, E. \& Cunha, D. de O. da. (2018). The importance of using COBIT model in IT processes in an organizational. Braz. J. of Develop. Curitiba, 4(6), 2844-2854.

Silva, H. B. G. \& Marques, R. M. (2018). Restrições ao Acesso Informacional: A Neutralidade de Rede e a Prática do Zero-Rating na Governança da Internet. Inf. \& Soc.:Est., 28(3), 169-182.

Silva, L. A. C., Araújo, M. V. P. \& Brito, M. L. A. (2018). Logística intermodal no estado do Rio Grande do Norte: um estudo exploratório. Research, Society and Development, 7, e477100.

Silva, S. E., Reis, L. P., Pereira, A. D. S. \& Fernandes, J. M. (2019). A Knowledge Taxonomy in The Context of Organizational Routines: An Study In a Public University. Inf. \& Soc.:Est., 29(2), 201-218.

Shleifer A., Vishny W. (1997). A Survey on Corporate Governance, The Journal of Finance, 52(2).

Schmitt, A., Raisch, S. \& Volberda, H. W. Strategic Renewal: Past Research, Theoretical Tensions and Future Challenges. International Journal of Management Reviews, v. 20, p. 81-98, 2018. DOI: 10.1111/ijmr.12117.

Steinberg, H. (2003). A dimensão humana da governança corporativa: pessoas criam as melhores e piores práticas. 2 ed. São Paulo: Gente.

Sommer, S. C., Loch, C. H. \& Dong, J. (2009). Managing Complexity and Unforeseeable Uncertainty in Startup Companies: An Empirical Study. Organization Science, 20(1),118-133. 10.1287/ orsc.1080.0369.

Tiwana, A. (2008). Do bridging ties complement strong ties? An empirical examination of alliance ambidexterity. Strategic Management Journal, 29(3), 251272. $10.1002 / \mathrm{smj} .666$.

Van Gremberger, W. (2004). Strategies for information technology governance. O Hershey, PA: Idea Group Publishing.

Vergara, S. C. \& Ferreira, V. C. P. (2005). A representação social de ONGs segundo formadores de opinião do Rio de Janeiro. Revista de Administração Pública, 39(5), 1137-1159.

Vergara, S. C. (2014). Projetos e Relatórios de Pesquisa em Administração. (15a ed.), Atlas.

Zattar, M. \& Marteleto, R. (2019). Information and Production of Knowledge Networks in The Governance of Water. Inf. \& Soc.:Est., $29,(1), 215-236$.

Zhang, Y. (1988). Definitions and Sciences of information. Information Processing \& Management, 24(4).

Zorrinho, C. (1995). Gestão da Informação. Condição para vencer. Iapmei, p.15.

Weill, P. (2004). O Segredo da Boa Governança. http://download.microsoft.com/download/7/d/f/7df01ca4-4dd4-42f4-9c1370a15d6e3450/MB32_Entrevista.p df, Microsoft Business.

Woida, L. M. (2020). Encontros Bibli: revista eletrônica de biblioteconomia e ciência da informação, 25, 01-24. Universidade Federal de Santa Catarina. 1518-2924. https://doi.org/10.5007/1518-2924.2020.e70464

Wu, S. P. J., Straub, D. W. \& Liang, T. P. (2015). "How information technology governance mechanisms and strategic alignment influence organizational performance: insights from a matched survey of business and it managers", MIS Quarterly, 39(2), 497-518. 